\title{
Mālama nā makua i nā keiki me ka hānō: Native Hawaiian Parents Car- ing for Their Children with Asthma
}

Asian/Pacific Island Nursing Journal

Volume 2(3): 74-82

(C)Author(s) 2017

http://digitalscholarship.unlv.edu/apin/

\author{
May K. Kealoha ${ }^{a}$ and Merle Kataoka-Yahiro ${ }^{b}$
}

\begin{abstract}
Native Hawaiian children have the highest prevalence rate of asthma among all ethnicities in the State of Hawai' $i$. Literature is limited regarding native Hawaiian parents' perception and experience caring for their children with asthma. The purpose of this study is to explore contemporary native Hawaiian parents' perspective and experience of caring for their children with asthma in the context of uncertainty. We applied a descriptive qualitative approach by means of directed content analysis using focus groups. Directed content analysis applied Mishel's Uncertainty in Illness Theory to guide data collection, organization, and analysis. We found that parents' personal stories about their children to be rich and enlightening. Findings verified that native Hawaiian parents experience uncertainty regarding asthma care as commonly described in the literature. Contextual influences including indigenous worldview and cultural values affected native Hawaiian parents' perceptions and experiences with conventional asthma care. Unique findings involved the etiology of asthma, features of social support ('ohana), and differentiation between Western medicine and traditional healing practices. As nurses focus on supporting the family's cultural values and preferences related to asthma care and alternative remedies, native Hawaiian parents' care of their children with asthma will be strengthened.
\end{abstract}

Keywords: native Hawaiian, culture, indigenous, childhood asthma, uncertainty, focus group, parents

While uncertainty is experienced by most parents in the care of their chronically ill children (Stewart \& Mishel, 2000), it is particularly true of parents of children with asthma (Chen, Huang, Yeh, \& Tsai, 2015). In the illness experience, uncertainty has four aspects: (a) ambiguity concerning the state of the illness, (b) complexity regarding treatment and system of care, (c) lack of information about the diagnosis and seriousness of the illness, and (d) unpredictability of the course of the disease and prognosis (Mishel, 1990).

The indigenous people of the Hawaiian Islands are known as kānaka maoli, native Hawaiians. Native Hawaiian children have the highest prevalence rate of asthma among children of all ethnicities in the State of Hawai' $i$. Their parents' indigenous worldview, cultural values, historical context, and effects of assimilation and acculturation may contribute to an experience of uncertainty regarding conventional asthma care. However, research is sparse regarding native Hawaiian parents' perspective and experience caring for children with asthma, particularly in the context of uncertainty.

\section{Background}

Asthma is one of the most prevalent chronic childhood diseases in the United States (Bloom, Cohen, \& Freeman, 2012). Nationally, 6.2 million children currently have asthma, representing $8.4 \%$ of children in the United States (Centers for Disease Control and Prevention, 2015). Hawai'i has one of the highest prevalence rates of childhood asthma in the United States (Wu, Cabana, Hilton, \& Ly, 2011), making the disease a major public health concern for the state (Hawai'i State Department of Health, 2014). Child asthma status for Hawai'i for years 2012 through 2015 ranged from $9.9 \%$ to $16 \%$ of the population (State of Hawai'i, Department of Health, 2017). Furthermore, an ethnicity-related disparity

\footnotetext{
${ }^{a}$ Kapi olani Community College, USA

${ }^{b}$ University of Hawai 'i at Mãnoa, USA
}

Corresponding Author:

May Kealoha, RN, MPH, MSN, PhD

Professor/Nursing Department

Kapi'olani Community College

Email: kealohab@hawaii.edu 
among children with asthma exists. Native Hawaiian children have significantly higher asthma prevalence $(19.4 \%)$ compared to the percentage of children of other ethnicities (White 7.1\%, Filipino 7.3\%, others 7.3\%; State of Hawai'i, Department of Health, 2017). Behavioral Risk Factor Surveillance System Asthma Call-Back Surveys identified native Hawaiian/Pacific Islander children with impaired asthma control to have high exposure to cockroaches but low exposure to tobacco smoke and mold (Wu et al., 2011).

Many parents have difficulty managing their child's asthma because of the multifaceted nature of the disease and treatment (Arcoleo, Zayas, Hawthorne, \& Begay, 2015). Nearly all parents of children with asthma display an uneven mastery of the complex processes of recognizing initial asthmatic symptoms, understanding the diagnosis, learning and implementing a treatment regimen, and finally, transferring their knowledge and skill to the affected child (Trent, Zimbro, \& Rutledge, 2015).

The only research involving native Hawaiian parents caring for their children with asthma, which the authors currently are aware of, was conducted by Tse and Palakiko (2004) using a community-based participatory approach. Their research found that parents relied heavily on emergency asthma medication and depended on direct physician involvement for care. Many were not aware of the seriousness of the chronic disease or when their child was having difficulty with the illness. There is a paucity of literature regarding native Hawaiian parents and their care of children with asthma.

\section{Statement of Problem and Research Question}

Native Hawaiians as indigenous people should have a voice in the development of asthma programs and be given opportunities to express what types of support and assistance would be of true benefit to them. Contextual influences including worldview, cultural values, history, and assimilation and acculturation factors may affect native Hawaiian parents' perceptions and experiences with conventional asthma care. New research is needed to provide contextual information in the effort to understand contemporary native Hawaiian parents' perspectives and experiences with asthma care. The research question was, what are native Hawaiian parents' perspective and experience of caring for their children with asthma in the context of uncertainty?

\section{Method}

\section{Design}

In this study, we applied a descriptive qualitative approach by means of directed content analysis using focus groups. Because studies on native Hawaiian parents' experience caring for children with asthma were limited, utilizing a theoretical guide for data collection and analysis ensured appropriate organization, collection of relevant data, and interpretation of findings (Canino et al., 2009). Mishel's Uncertainty in Illness Theory (UIT), a well-established nursing theory based on the concept of uncertainty, served as the study's theoretical framework (Mishel, 1990). The framework was broad and able to incorporate contextual features such as indigenous culture, cultural practices, and health beliefs (Walker \& Avant, 2011). The UIT specifically contained constructs and categories that concentrated on the antecedents of uncertainty, modulating factors that increased or decreased uncertainty, and described attributes and implications of uncertainty (Mishel, 1990). A comprehensive assessment and understanding of parents' uncertainty experience were completed by utilizing the UIT.

Focus groups for data collection in this study allowed Hawaiian parents to respond to openended questions in their own words and from their unique perspectives regarding health, beliefs, values, practices, cultural interpretations, and cultural insights (Bernard \& Ryan, 2010; Castleden et al., 2016). We conducted the focus groups in the informal "talk story" format, an acceptable form of gaining information from native Hawaiians (Andrade, 2008). The focus group questions adhered to three major guidelines: (1) respect cultural etiquette and practice of asking questions, sharing information, and applying Hawaiian words and concepts (Andrade, 2008); (2) apply focus group process of asking broad questions followed by more specific questions (Krueger \& Casey, 2009); and (3) align with the constructs of the UIT (Mishel, 1990). The initial questions were reviewed and revised by three native Hawaiian consultants familiar with asthma and/or having experience caring for their child with asthma. Then a pilot study was conducted with one group of parents meeting the inclusion criteria. The final eight open-ended questions were (1) When did it (illness/asthma) start? (2) What do you do to care for your child with asthma? (3) What things or people have made it easier for you as a parent caring for your child with asthma? (4) What things have made it harder for you as a parent? (5) What is it like for you as a parent taking care of your child? (6) How do you manage caring for your child with asthma? (7) How has your Hawaiian culture influenced you in the care of your child? (8) What matters most to you as a parent about this illness or treatment?

\section{Sample}

A purposive sampling of native Hawaiian parents with children with asthma was recruited with no restrictions regarding age, socioeconomic status, 
gender, or number of children to obtain a wide range of perspectives. The inclusion criteria of eligible parent participants were (a) self-reports as of Hawaiian ancestry, (b) is a biological parent, (c) over 18 years of age, (d) self-reports no cognitive or emotional impairment, (e) has a child aged 18 years or younger, and (f) reports child has had current asthma for one year or more. We specifically recruited biological parents as there may be multiple parental roles in families. The asthma history of one year or more confirmed the requisite for parents to have experience with the illness.

\section{Recruitment}

Recruitment of native Hawaiian participants involved distributing informational flyers, gaining permission from organizational leaders to directly contact prospective participants, and recruiting key informants to identify prospective participants and linking them with the researcher (Krueger \& Casey, 2009). Informal communication by "word of mouth" was a culturally appropriate method of recruitment, rather than using standard methods such as radio announcements and advertisements (Chang, 2001).

Although 17 parents met the inclusion criteria and considered prospective participants, seven parents were unable to attend the scheduled session due to illness, birth, or a conflicting work schedule. Two interested adults did not meet the inclusion criteria due to the child's age and not being the biological parent. As a result, there was a total of 10 participants. Four focus group sessions included three participants in Group 1, one participant in Group 2 (two prospective participants did not attend), two participants in Group 3, and four in Group 4. Nine participants resided in Ko'olau Poko (Windward) and one in Honolulu, O`ahu. We offered each participant incentives of $\$ 50$ and two bags of poi (pounded cooked taro), a highly valued cultural food. The settings were at community college classrooms and selected based on convenience, accessibility, freedom from interruptions, and sense of safety or neutrality for participants.

\section{Data Collection}

The research team consisted of a principal investigator and research assistant. Data sources included a demographic survey and focus group discussions. Following approval from the University of Hawai'i at Mānoa, Human Studies Program (CHS\#19503), data collection occurred from October 2011 to January 2012. We implemented a protocol or focus group procedure for each focus group to maintain consistency and order; the procedure ensured participant attendance and complete set-up of the physical facility. Informed consents were obtained before the start of the session; we allotted two hours for each focus group.

\section{Data Analysis}

Data analysis included descriptive analysis of the demographic data and direct content analysis of the focus group sessions. We collected data from the focus group sessions from three sources: (a) individual participants' responses, (b) responses that occurred due to the interaction of the participants as they heard and responded to each other, and (c) consensual and differing group opinions (Houser, 2012; Krueger \& Casey, 2009). Through directed content analysis, data were investigated to understand, digest, synthesize, conceptualize, and re-conceptualize descriptions of feelings, behaviors, experiences, and ideas (Hsieh \& Shannon, 2005; Olsson, Eriksson, \& Anderzén-Carlsson, 2017; Presseau et al., 2017). Subsequently, interpretive coding of the data was conducted to identify constructs, categories, subcategories, and new themes (Houser, 2012; Presseau et al., 2017).

Codes of constructs, categories, and subcategories, and their relationships were predetermined by the UIT (Hsieh \& Shannon, 2005; Olsson et al., 2017; Presseau et al., 2017). UIT constructs consisted of the Stimuli Frame, Cognitive Capacities, Structure Providers, Uncertainty, Appraisal, and Adaptation. Categories were branches or offshoots of constructs. Subcategories became more specific dimensions of categories. If the UIT directed codes of constructs, categories, and subcategories did not reflect data, then alternative codes were created to identify new themes (Hsieh \& Shannon, 2005; Presseau et al., 2017 ). Content analysis was first performed by the research team members manually then data was entered into NVivo (NVivo, 2011).

We established key ideas that frequently reflected the meaning of the data through the process of construct and category identification and comparison of data (Burns \& Grove, 2011). Eventually, information appeared redundant with no new information gained. Each UIT construct, category, and subcategory contained applicable coded data. After Group 4 transcripts were reviewed and coded, the research team including an external reviewer determined by consensus that data saturation was achieved and data collection ceased.

\section{Assurance of Rigor}

We maintained rigor by a strict process of data collection and analysis. Aspects of rigor in qualitative research included (a) trustworthiness of the data collected (initial testing of questions by native Hawaiian consultants, validating findings with partic- 
ipants), (b) confirmability (consistency and ability to duplicate decision making regarding data collection and data analysis were enhanced through the use of NVivo), and (c) credibility of the findings (having summaries reviewed by participants through "member checks"; Houser, 2012). We assured reliability of the findings through internal consistency and interrater reliability of the coding system by the research team members. We also completed an external check by an external reviewer who independently analyzed the data (Bernard \& Ryan, 2010).

\section{Results}

\section{Demographic Characteristics}

There were a total of 10 participants: nine females and one male. The majority of participants were married. All parents completed high school, and $40 \%$ of parents had greater than a high school education. The ages of the parents ranged from 22 to 56 years, with a mean age of 30 years. Most parents (70\%) were employed. All of the children had medical insurance and an identified health care provider. Four (40\%) received Medicaid insurance, and six $(60 \%)$ had private health insurance.

Each parent was asked to answer questions based on one child with asthma (total of 10 children). Most of the children were male $(n=8)$ and the eldest child. Children's age groups included one (10\%) toddler, three $(30 \%)$ preschoolers, five $(50 \%)$ in elementary, and one (10\%) in high school. Six $(60 \%)$ children were the only child in the family with asthma. Four $(40 \%)$ children had one sibling with asthma.

\section{Uncertainty in Illness Theory}

The study findings indicated that native Hawaiian parents experience uncertainty while caring for their child with asthma. Each UIT construct, category, and subcategory were individually addressed and supported by significant statements and quotes made by the participants. Four new subcategories (Asthma Experience, Asthma Triggers, Direct actionWestern, and Complementary and Alternative Medicine) emerged from the study results.

Construct: Stimuli Frame, Category: Event Familiarity, Subcategory Asthma Experience

The asthma experience subcategory identified parents with limited asthma experience as having more uncertainty than parents with many years of asthma management. Those without experience $(n=$ 3 ; 30\%) reported much confusion and uncertainty about asthma symptoms, asthma triggers, and asthma management. One participant described her confusion by saying, "... her doctor said to give it [medication and treatment] to her [daughter] as needed. But I didn't know when she needed it. When does she need it? When she coughs? I don't know."

Construct: Stimuli Frame, Category: Event Familiarity, Subcategory Asthma Triggers

Asthma triggers emerged as a new subcategory because unidentified triggers and unpredictable effects of asthma triggers were strong antecedents to uncertainty for parents. One participant described the wide range of triggers that make living in Hawai'i difficult for people with asthma.

And we live in Hawai' $i$. There is [are] cockroaches everywhere so you know if my daughter has her asthma ..." Oh no! We are not going [there]. There is [are] a lot of cockroaches." [Laughter] It is everything ... especially living in Hawai' $i$ with the Kona winds and the vog [volcanic air pollution] and the weather changes drastically from sunny to rainy and just the steam that comes off of the pavement after it rains or the flowers or perfume ....

\section{Construct: Cognitive Capacities}

Multiple socioeconomic factors adversely affected the parents' capacity to process information about asthma. One participant reported, "It was just a little overwhelming for me... not being able to be there for him all the time .... being at school and work, I can't think sometimes. I'm like, oh my goodness, what am I going to do?"

Construct: Structure Providers, Category: Social Support

'Ohana served as the participants' primary social support and assisted parents by decreasing their uncertainties about asthma symptom patterns and asthma management by providing information, service-related assistance (child care, meal preparation, medication administration, transporting child to doctor's office), and emotional support. 'Ohana consists of a large circle of nuclear and extended family members including spouses, mothers, fathers, sisters, grandmothers $(t \bar{u} t \bar{u})$, grandfathers, aunts, uncles, and cousins. Many tūtū and küpuna [elders] assisted parents with asthma care by performing traditional healing practices. Parents demonstrated traditional respect for küpuna by expressing their appreciation for any asthma advice or assistance offered. In this way, cultural values of lokkhi [harmony] and pono [proper and good] were maintained. Parents reported accepting $t \bar{u} t \bar{u}$ remedies, applying some and storing others away.

Not all members of parents' social network were sympathetic. One parent felt criticized because family members would ask, "Why is he sick again? 
What are you doing?" Most fathers/husbands reportedly "cannot handle" seeing their child in distress and preferred that mothers implement asthma treatment.

\section{Construct: Uncertainty, Lack of Information}

Parents were interested in understanding why so many native Hawaiians had asthma and wondered how their children acquired asthma. One parent commented, "It's just frustrating. What is it about Hawaiians that trigger this asthma?" A father stated, "[Asthma] could be one haole [foreign person] thing. I don't know." Another member agreed by saying, "I think so. Because it [asthma] wasn't so prevalent back then."

Most participants practiced information seeking. One focus group identified the need for a central repository containing up-to-date, pertinent asthma information in the form of a website that is "a local style asthma control ... just something that really defines Hawai' $i$ " and having an 'asthma dictionary' that contains remedies including those from other cultures such as Chinese teas." Designing an asthma information page like the immunization fact sheet parents receive before the administration of a vaccine was recommended by one parent. She suggested, "Something you can read while you are waiting for the physician to come in."

Participants explained that conventional asthma health education and teaching strategies were not engaging. They preferred learning by informally sitting with a small group of parents in one central place where "you can go to and people share their mana' $o$ [thoughts, ideas], they share their stories, they share what works and what doesn't work and with families .... I think that would help us a lot in Hawai'i."'

\section{Construct: Appraisal: Inference of Danger,} Category: Coping Mobilizing Strategies, Direct Action - Western, Complementary and Alternative Medicine (CAM)

UIT coping strategies included four subcategories: vigilance, information seeking, direct action, and Western and complementary and alternative medicine (CAM). Parents were vigilant in monitoring for asthma symptoms and asthma triggers and sought information about asthma from health professionals, 'ohana, and the internet. The difference between Western and CAM therapies was a new cultural finding. All participants reported administering prescribed medical treatment (medication, aerosol treatment) for respiratory distress. Six parents referred to prescribed medical treatment as "Western" therapy, "more Western like style," and "very Western medicine."

Parents offered three types of CAM therapies that were identified and categorized by the re- search team: (1) comfort measures, (2) home remedies, and (3) Hawaiian cultural healing practices. Comfort measures included stopping their child from running, having them sit and relax, asking them to take deep calming breaths, and "mist in tub." One parent described home remedies as "backyard style of doing things and ... their own non-Western way of doing things and treating." The remedies were "handed down" to the mothers by their tūtu and included applying Vicks to one's chest, back, and/or underarms; "towel over the head," eucalyptus oil "under their feet, on their chest," offering hot water or warm tea, placing vaporizers in rooms, and having children consume caffeinated drinks such as soda and coffee.

$T \bar{u} t \bar{u}$ reportedly believed in superstitions, curses, teas, applying warm, dry compresses, and lomi [massage]. Other traditional healing practices included eating poppolo (Solanum nigrum) berries, boiling pōpolo berry leaves for tea, applying ti (Cordyline terminalis) leaf to the chest, placing cold ti leaf to the head for fever, drinking Hawaiian tea and noni (Morinda citrifolia), and prayer. A parent explained, "... with lomi in your family you learn... how to bring your child back down to a stable level. Because when they are with asthma, they are either shooting up with energy or they are so drained of energy ..." Another participant recalled the relevance of the ocean to healing for native Hawaiians. Her küpuna had said, "Take him to the beach, let him go swim in the salt water. Dry it all out."

The actual application of native Hawaiian cultural healing practices among participants was limited. Four participants were not exposed to healing traditions for asthma or respiratory symptoms. Two parents recalled the former prohibition of native Hawaiian cultural practices. One parent explained, "With the culture, the language, the art was banned. It was taboo. A lot of our kuppuna didn't pass it [healing practices] down to their kids so we've kind of adopted the modern way."

A different participant explained her rationale for using comfort measures and Hawaiian practices before administering medication:

I think being Hawaiian and being a part of our culture you learn different things ... out of the Western way, out of the medicine. Because you don't always want your kid on medicine. I don't want my daughter to be reliant on it [asthma medication] ... if at all possible I would rather stick to natural resources and other mechanisms before I give her the albuterol and what-not. And I definitely will ... do [CAM therapy] ... first.

Coping strategies that parents used to reduce uncertainty and control emotional responses included methods of faith and comparing the patient's condition to others who were worse. Those parents who were not heavily engaged with their children's asth- 
ma care reportedly exercised more buffering coping strategies than the primary caregivers. Some parents also continued to have hopes of a cure and healing from asthma. Three parents believed that physical activity would help their children "grow out of asthma." Five fathers reportedly utilized avoidance and disengagement by going to work and/or leaving the child with the mother to administer asthma care.

\section{Construct: Adaptation}

Positive adaptation involved goal directed behaviors that promoted the acquisition of developmental milestones and engagement in normal physical activities. Families of older children demonstrated more goal directed adaptation than those with younger children. Adequate financial and social resources may have contributed to this difference. All parents experienced biopsychosocial adaptation difficulties. Parents reported lack of sleep due to worry and ongoing caretaking responsibilities.

\section{Discussion}

The results of this study suggest that native Hawaiian parents' perception, knowledge, and coping with the uncertainty of asthma care is related to a lack of information, unpredictability of asthma exacerbations, prognosis, and complexity of treatment. The participants in the study expressed three concerns about the etiology of asthma not found in the literature: (a) its foreign origin, (b) native Hawaiians being more susceptible to the condition, and (c) the effect of "vog" as a trigger.

Health care professionals are the foremost source of information, guidance, and support regarding asthma management for the study participants. Some parents in the study reported difficulty understanding the provider's instructions and explanations. Similar findings in the literature related to provider characteristics and expectations, stereotyping of parents, and use of ineffective communication skills (Hines, 2011). Notably, when providers demonstrated personalismo [a warm and personal approach], Latino parents were more inclined to trust what was being communicated (Desai, Rivera, \& Backes, 2016). Like personalismo, aloha [compassion] and kōkua [helpfulness] are equally significant to Hawaiian parents in receiving information (The Native Hawaiian Health Research Consortium, 1985). There was an association between characteristics of the provider (friendliness) and the quality of their relationship (trusting, on-going) and high satisfaction of clinic visits by parents of children with chronic diseases (Swedlund, Schumacher, Young, \& Cox, 2012).

The concept of 'ohana is central to the Hawaiian culture and social system (Handy \& Pukui, 1998). It is similarly found in other cultures such as
Latino (la familia; Desai et al., 2016), Mexican (familismo; Arcoleo et al., 2015), and Māori (whānau; Jones, Ingham, Cram, Dean, \& Davies, 2013). In these cultures, the family is the primary source of social relationships and assistance crucial to one's sense of identity and self-worth (Desai et al., 2016; Jones et al., 2013). Family support (Scheckner, Arcoleo, \& Feldman, 2015) and higher levels of family connectedness and stronger ethnic identity appear to be protective factors against asthma-related limitations (Koinis-Mitchell et al., 2012). Family group orientation is customary in Asian cultures (Purnell, 2014) as well as indigenous peoples such as Native Americans (McCubbin, Thompson, Thompson, McCubbin, \& Kaston, 1993) and Aboriginal people of Canada (Castleden et al., 2016).

Emotional and service-related support from partners, extended family, and friends were common findings in the literature (Cashin, Small, \& Solberg, 2008; Desai et al., 2016). However, the 'ohana in the study appeared to be a much larger network of involved relatives than listed in the literature (Cashin et al., 2008). Fathers were actively engaged in their child's asthma management in several research studies (Cashin et al., 2008; Swallow, Macfadyen, Santacroce, \& Lambert, 2011) but not reported in this study. Future studies in this area need to include the paternal role and perspective in caring for a child with asthma.

In addition to 'ohana support, one participant suggested having a place for native Hawaiian parents to share their stories and mana' $o$ about asthma. This suggestion for support groups is in alignment with indigenous communities' philosophy of a "community-raised child" (Castleden et al., 2016). Other indigenous people such as the Mi'kmaq have expressed strong interest in the development of support groups that will provide a larger network of community help and understanding (Castleden et al., 2016). One focus group of tribal Native American participants recommended having asthma education be delivered at monthly family dinners and sporting events "because tribal communities are familyoriented, and have many community events" (Rodehorst-Weber, Wilhelm, Stepans, Tobacco, \& delaPaz, 2009).

In the face of uncertainty, the findings in this study and existing literature described several coping strategies used by participants such as vigilance (Cashin et al., 2008) and information seeking (Cashin et al., 2008; Desai et al., 2016; Swallow et al., 2011). Also, the study identified two types of therapies utilized by native Hawaiians: Western therapies and CAM remedies. In the literature, families relied on CAM in the form of home remedies and folk medicine in place of or in addition to conventional medications because (a) of their cultural and 
health belief system (Arcoleo et al., 2015; Desai et al., 2016), (b) concern about the side effects and long-term effects of asthma medication (Scheewe et al., 2011), and/or (c) high cost of medical therapies (Shen \& Oraka, 2012). According to Shen and Oraka (2012), $26.7 \%$ of 5,435 children with current asthma reported CAM use in the Asthma Call Back Survey. CAM therapies included breathing techniques, vitamins, herbal products, aromatherapy, homeopathy, and massage (Shen \& Oraka, 2012). It should be noted that parents may not openly discuss their use of traditional health practices to health care professionals because they are fearful of ridicule or reproof (Desai et al., 2016).

Not all the study participants used CAM therapies. The diminishing application of traditional Hawaiian healing practices and increasing use of Western products may be due to assimilation and acculturation of Western culture, increasing time constraints and preference for quick medical therapies, lack of information about traditional healing practices, and insufficient traditional experts and küpuna to serve as resources (Chang, 2001; Chun, 2012). Literature corroborates a similar situation for Native Americans. Traditional approaches to treat breathing difficulties for Native Americans still exist (Rodehorst-Weber et al., 2009). But such practices are uncommon in certain tribal groups because of colonization, living off traditional lands, discontinuance of cultural practices, and lack of accessible traditional practitioners due to geographical barriers (Battiste, 2000).

Adaptation to asthma challenges was related to the constant and overwhelming responsibility for asthma management (Arcoleo et al., 2015; Silva, Carona, Crespo, \& Canavarro, 2015). Parents with more experience managing asthma recognized asthma symptoms and triggers confidently determined the next course of action over a failed therapy and utilized the health care system to their benefit (Arcoleo et al., 2015). Parents with young children with asthma and limited asthma experience reported more uncertainty regarding the disease condition and its management (Desai et al., 2016; Silva et al., 2015; Silva, Crespo, Carona, \& Canavarro, 2015). Additional socioeconomic difficulties identified in the literature included securing competent babysitters, working, being absent at work, jeopardizing ones' employment, experiencing financial stress, not finding adequate housing, adopting disorganized family routines, lacking sleep, and experiencing fatigue (Carrillo et al., 2015; Goedken, Urmie, \& Polgreen, 2015; Hines, 2011; Krieger, Philby, \& Brooks, 2011).

\section{Limitations}

The limitation of this study is that it cannot be generalized to native Hawaiian and non-native
Hawaiian parents who have children with asthma. The participants were primarily from Ko'olau Poko, $\mathrm{O}^{\prime}$ ahu and their responses reflect this locale or sense of place (Oneha, 2001). The findings, however, provide information about an area where there is so little research done, but of which there are great health disparities. This study will contribute and add to the body of existing literature on asthma care and management of native Hawaiian parents and their children with asthma.

\section{Conclusions}

The personal stories of native Hawaiian parents caring for their children have been rich and revealing. The study's findings verified that contemporary native Hawaiian parents experience uncertainty regarding asthma care as commonly described in the literature. Contextual influences including indigenous worldview and cultural values affected their perceptions and experiences with conventional asthma care. As nurses focus on supporting the family's cultural values and preferences related to asthma care and alternative remedies, native Hawaiian parents' care of their children with asthma will be strengthened.

\section{Acknowledgment}

The first author would like to acknowledge her dissertation committee members: Drs. Merle Kataoka-Yahiro, Lana Sue Ka'opua, Karol Richardson, Karen Tessier, \& Lorieana Yancura; and research assistants: Drs. Sandra Sinclair \& Hatsumi Taniguchi.

\section{Declaration of Conflicting Interests}

The authors declare no conflicts of interest.

\section{References}

Andrade, C. (2008). Hā'ena: Through the eyes of the ancestors. Honolulu, HI: University of Hawai'i Press.

Arcoleo, K., Zayas, L. E., Hawthorne, A., \& Begay, R. (2015). Illness representations and cultural practices play a role in patient-centered care in childhood asthma: Experiences of Mexican mothers. Journal of Asthma, 52, 699-706. https://doi.org/10.3109/02770903.2014.1001905

Battiste, M. (2000). Reclaiming indigenous voice and vision. Vancouver: UBC Press.

Bernard, H. R., \& Ryan, G. W. (2010). Analyzing qualitative data: Systematic approaches. Los Angeles, CA: SAGE.

Burns, N., \& Grove, S. (2011). Understanding nursing research: Building an evidenced-based practice (5th ed.). Maryland Heights, MO: Elsevier Saunders. 
Bloom, B., Cohen, R. A., \& Freeman, G. (2012). Summary health statistics for U.S. children: National Health Interview Survey, 2011. Vital and Health Statistics, 10(254), 1-88. Retrieved from https://www.cdc.gov/nchs/data/series/sr_10/sr10 250.pdf

Canino, G., McQuaid, E. L., Alvarez, M., Colon, A., Esteban, C., Febo, V., ... Fritz, G. (2009). Issues and methods of disparities research: The Rhode Island-Puerto Rico asthma center. Pediatric Pulmonology, 44, 899-908. https://doi.org/10.1002/ppul.21075

Carrillo, G., Han, D., Lucio, R., Seol, Y., Chong-Menard, B., \& Smith, K. (2015). Impacting environmental and public health through the use of dual targeted and tailored asthma educational interventions. Journal of Environmental and Public Health. https://doi.org/10.1155/2015/476173

Cashin, G. H., Small, S. P., \& Solberg, S. M. (2008). The lived experience of fathers who have children with asthma: A phenomenological study. Journal of Pediatric Nursing, 23, 372-385. https://doi.org/10.1016/j.pedn.2007.08.001

Castleden, H., Watson, R., Bennett, E., Masuda, J., King, M., Stewart, M., \& Tui'kn Partnership. (2016). Asthma prevention and management for Aboriginal people: Lessons from Mi'kmaq communities, Unama'ki, Canada, 2012. Preventing Chronic Disease, 13. https://doi.org/10.5888/pcd13.150244

Centers for Disease Control and Prevention (CDC). (2015). Summary health statistics: National Health Interview Survey, 2015. Retrieved from https://www.cdc.gov/nchs/fastats/asthma.htm

Chang, H. (2001). Hawaiian health practitioners in contemporary society. Pacific Health Dialog, 8, 260-273. Retrieved from http://pacifichealthdialog.org.fj/index_option_co m_content_view_article_id_69.html

Chen, S., Huang, J., Yeh, K., \& Tsai, Y. (2015). The stress of caring for children with asthma: A qualitative study of primary caregivers. Journal of Nursing Research. https://doi.org/10.1097/jnr.0000000000000083

Chun, M. N. (2012). Kāhuna: Traditions of Hawaiian medicinal priests and healing practitioners. Honolulu, HI: First People's Productions.

Desai, P. P., Rivera, A. T., \& Backes, E. M. (2016). Latino caregiver coping with children's chronic health conditions: An integrative literature review. Journal of Pediatric Health Care, 30, 108-120. https://doi.org/10.1016/j.pedhc.2015.06.001

Goedken, A., Urmie, J., \& Polgreen, L. (2015). Provider visits for asthma: Potential barriers for insured children. Global Journal of Health Science, 7, 96-105. https://doi.org/10.5539/gjhs.v7n5p96

Handy, E. S. C., \& Pukui, M. K. (1998). The Polynesian family system in Ka' $u$ Hawai' $i$. Honolulu, HI: Mutual Publishing.

Hawai'i State Department of Health. (2014). Hawaii coordinated chronic disease framework. Retrieved from http://health.hawaii.gov/chronicdisease/files/2014/09/CDFrameworkLR.pdf
Hines, A. B. (2011). Asthma: A health disparity among African American children. Journal of Pediatric Nursing, 26, 25-33. https://doi.org/10.1016/j.pedn.2009.10.006

Houser, J. (2012). Nursing research: Reading, using, and creating evidence ( 2 nd ed.). Boston, MA: Jones and Bartlett Learning.

Hsieh, H-F., \& Shannon, S. E. (2005). Three approaches to qualitative content analysis. Qualitative Health Research, 15, 1277-1288. https://doi.org/10.1177/1049732305276687

Jones, B., Ingham, T. R., Cram, F., Dean, S., \& Davies, C. (2013). An indigenous approach to explore health-related experiences among Māori parents: The Pukapuka Hauora asthma study. BMC Public Health, 13. https://doi.org/10.1186/1471-245813-228

Koinis-Mitchell, D., McQuaid, E. L., Jandasek, B., Kopel, S. J., Seifer, R., Klein, R. B., ... Fritz, G. K. (2012). Identifying individual, cultural and asthma-related risk and protective factors associated with resilient asthma outcomes in urban children and families. Journal of Pediatric Psychology, 37, 424-437. https://doi.org/10.1093/jpepsy/jss002

Krieger, J. W., Philby, M. L., \& Brooks, M. Z. (2011). Better home visits for asthma: Lessons learned from the Seattle-King county asthma program. American Journal of Preventive Medicine, 41(2 Suppl 1), S48-51. https://doi.org/10.1016/i.amepre.2011.05.010

Krueger, R. A., \& Casey, M. A. (2009). Focus groups: A practical guide for applied research. Thousand Oaks, CA: SAGE.

Mishel, M. H. (1990). Reconceptualization of the uncertainty in illness theory. Image: Journal of Nursing Scholarship, 22, 256-262. https://doi.org/10.1111/j.15475069.1990.tb00225.x

McCubbin, H. I, Thompson, E. A., Thompson, A. I., McCubbin, M. A., \& Kaston, A. J. (1993). Culture, ethnicity, and the family: Critical factors in childhood chronic illnesses and disabilities. Pediatrics, 91, 1063-1070.

NVivo. (2011). NVIVO 8 Getting Started. Retrieved from www.qsrinternational.com

Purnell, L. (2014). Guide to culturally competent health care (3rd ed.). Philadelphia, PA: F. A. Davis Company.

Presseau, J., Mutsaers, B., Al-Jaishi, A., Squires, J., McIntyre, C. W., Garg, A. X., \& Grimshaw, J. M. (2017). Barriers and facilitators to healthcare professional behaviour change in clinical trials using the Theoretical Domains Framework: A case study of a trial of individualized temperature-reduced haemodialysis. BioMedCentral Trials, 18. https://doi.org/10.1186/s13063-017-1965-9

Olsson, E., Eriksson, M., \& Anderzén-Carlsson, A. (2017). Skin-to-skin contact facilitates more equal parenthood - A qualitative study from father's perspective. Journal of Pediatric Nursing, 34, e29. https://doi.org/10.1016/j.pedn.2017.03.004 
Oneha, M. F. (2001). Ka mauli o ka 'oina a he mauli kanaka: An ethnographic study from an Hawaiian sense of place. Pacific Health Dialog, 8, 299311.

Rodehorst-Weber, T. K., Wilhelm, S. L., Stepans, M. B. F., Tobacco, R., \& delaPaz, F. (2009). Screening Native American children for asthma: Findings from focus group discussions. Issues in Comprehensive Pediatric Nursing, 32, 200-209. https://doi.org/10.3109/01460860903281382

Silva, N., Carona, C., Crespo, D., \& Canavarro, M. (2015). Caregiving burden and uplifts: A contradiction or a protective partnership for the quality of life of parents and their children with asthma? Journal of Family Psychology, 29, 151-161. https://doi.org/10.1037/fam0000071

Silva, N., Crespo, C., Carona, C., \& Canavarro, M. C. (2015). Mapping the caregiving process in paediatric asthma: Parental burden, acceptance and denial coping strategies and quality of life. Psychology \& Health, 30, 949-968. https://doi.org/10.1080/08870446.2015.1007981

Shen, J., \& Oraka, E. (2012). Complementary and alternative medicine (CAM) use among children with current asthma. Preventive Medicine, 54, 27-31. https://doi.org/10.1016/j.ypmed.2011.10.007

Swallow, V., Macfadyen, A., Santacroce, S. J., \& Lambert, H. (2011). Fathers' contribution to the management of their child's long-term medical condition: A narrative review of the literature. Health Expectations, 15, 157-175. https://doi.org/10.1111/j.13697625.2011.00674.x

Swedlund, M. P., Schumacher, J. B., Young, H. N., \& Cox, E. D. (2012). Effect of communication style and physician-family relationships on satisfaction with pediatric chronic disease care. Health Communication, 27, 498-505. https://doi.org/10.1080/10410236.2011.616632

Scheewe, S., Vogt, L., Minakawa, S., Eichmann, D., Welle, S., Stachow, R., \& Banzer, W. (2011). Acupunc ture in children and adolescents with bronchial asthma: A randomised controlled study. Complementary Therapies in Medicine, 19, 239-246. https://doi.org/10.1016/j.ctim.2011.07.002

Scheckner, B., Arcoleo, K., \& Feldman, J. M. (2015). The effect of parental support and acculturation on childhood asthma control. Journal of Asthma, 52, 606-613. https://doi.org/10.3109/02770903.2014.991969

State of Hawai'i, Department of Health. (2017). The Hawai'i Behavioral Risk Factor Surveillance System. Retrieved from http://health.hawaii.gov/brfss/

Stewart, J. L., \& Mishel, M. H. (2000). Uncertainty in childhood illness: A synthesis of the parent and child literature. Scholarly Inquiry for Nursing Practice, 14, 299-319. Retrieved from https://www.highbeam.com/publications/scholarl y-inquiry-for-nursing-practice-p166413

The Native Hawaiian Health Research Consortium. (1985). E ola mau: The Native Hawaiian health needs study: Medical task force report. Retrieved from http://www.papaolalokahi.org/images/pdffiles/91985-E-Ola-Mau-The-Native-HawaiianHealth-Needs-Study-Mental-Health-Task-ForceReport.pdf

Trent, C. A., Zimbro, K. S., \& Rutledge, C. M. (2015). Barriers in asthma care for pediatric patients in primary care. Journal of Pediatric Health Care, 29 , 70-79. https://doi.org/10.1016/j.pedhc.2014.07.002

Tse, A. M., \& Palakiko, D. (2004). Perceptions of barriers and facilitators to pediatric asthma management in Native Hawaiian children: Final Report. Honolulu, HI: University of Hawaii - Mānoa.

Walker, L. O., \& Avant, K. C. (2011). Strategies for theory construction in nursing. Boston, MA: Prentice Hall.

Wu, B. H., Cabana, M. D., Hilton, J. F., \& Ly, N. P. (2011). Race and asthma control in the pediatric population of Hawaii. Pediatric Pulmonology, 46, 442-451. https://doi.org/10.1002/ppul.21387 